\title{
A complexidade do reconhecimento de grafos $k$-fino próprio de precedência
}

\author{
Flavia Bonomo-Braberman ${ }^{1}$, Fabiano S. Oliveira ${ }^{2 *}$, Moysés S. Sampaio Jr. ${ }^{3 \dagger}$ \\ Jayme L. Szwarcfiter ${ }^{2,3 \ddagger}$ \\ ${ }^{1}$ DC-FCEN/ICC-CONICET, Universidad de Buenos Aires, Argentina \\ ${ }^{2} \mathrm{IME}$ - Universidade do Estado do Rio de Janeiro (UERJ), Brasil \\ ${ }^{3}$ COPPE/PESC - Universidade Federal do Rio de Janeiro (UFRJ), Brasil \\ fbonomo@dc.uba.ar, fabiano.oliveira@ime.uerj.br, [moysessj,jayme]@cos.ufrj.br
}

\begin{abstract}
The class of precedence proper k-thin graphs is a subclass of proper $k$-thin graphs. The complexity status of recognizing proper $k$-thin graphs is unknown for any fixed $k \geq 2$. In this work, we prove that, when $k$ is part of the input, the problem of recognizing precedence proper $k$-thin graphs is NPcomplete, and we present a characterization for them based on threshold graphs.
\end{abstract}

\section{Introdução}

Um grafo de intervalo $G$ é tal que $V(G)$ corresponde a uma família de intervalos fechados distintos da reta real, chamada de modelo, e $(I, J) \in E(G)$ se, e somente se, $I \cap J \neq \emptyset$. Um grafo de intervalo é denominado próprio se admitir ao menos um modelo tal que $I \nsubseteq J$, para todo $I, J \in V(G)$. Em [Roberts 1969] é mostrado que um grafo é de intervalo próprio se, e somente se, existir uma ordem total $s$ de $V(G)$ tal que, para qualquer tripla $(p, q, r)$ de vértices ordenada com respeito a $s$, se $(p, r) \in E(G)$ então $(p, q),(q, r) \in$ $E(G)$. Tal ordem $s$ é denominada canônica própria. A Figura 1 mostra um grafo de intervalo próprio e uma de suas ordens canônicas próprias: $c<b<d<a<e<f$.

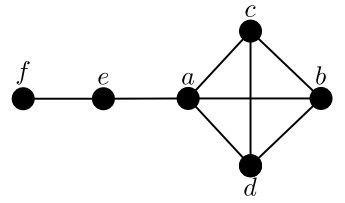

(a)

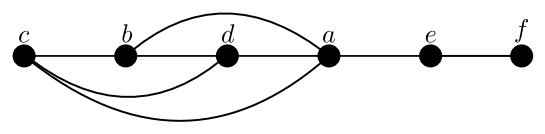

(b)

Figura 1. (a) Grafo de intervalo próprio $G$ e (b) Ordem canônica própria de $G$.

Um grafo $G$ é dito k-fino próprio [Bonomo and de Estrada 2019] se existirem uma ordem total $s$ e um $k$-particionamento de $V(G)$ tais que, para qualquer tripla ordenada $(p, q, r)$ em relação a $s$, tem-se que (i) se $p$ e $q$ pertencerem a uma mesma parte e $(p, r) \in E(G)$, então $(q, r) \in E(G)$ e (ii) se $q$ e $r$ pertencerem a uma mesma parte e $(p, r) \in E(G)$, então $(p, q) \in E(G)$. Uma ordem com essa propriedade é chamada de ordem fortemente consistente. Grafos $k$-fino próprio generalizam grafos

* parcialmente financiado por FAPERJ.

${ }^{\dagger}$ financiado por CAPES.

${ }^{\ddagger}$ parcialmente financiado por FAPERJ e CNPq. 
de intervalo próprio, sendo os grafos 1-fino próprio a classe dos grafos de intervalo próprio. Em [Bonomo and de Estrada 2019], é provado que, dado um particionamento de tamanho arbitrário de $V(G)$, decidir a existência de uma ordem fortemente consistente com relação a esse particionamento é um problema NP-completo. Por outro lado, a complexidade da versão desse problema quando o tamanho do particionamento é fixo, isto é, não faz parte dos dados de entrada, é um problema ainda em aberto. Ainda em [Bonomo and de Estrada 2019], é mostrado que dada uma ordem total de $V(G)$, determinar o particionamento mínimo para o qual essa ordem é fortemente consistente pode ser resolvido em tempo polinomial. Além disso, considerando o caso geral, determinar se um grafo é $k$-fino próprio está em aberto mesmo para um $k \geq 2$ fixo.

Neste trabalho, abordamos uma classe mais restrita de grafos $k$-fino próprio, na qual só são permitidas ordens fortemente consistentes com uma determinada propriedade. Um grafo $k$-fino próprio de precedência $(k-F P P)$ [Oliveira et al. 2018] é um grafo $k$-fino próprio que admite uma ordem fortemente consistente em que os vértices que pertencem a uma mesma parte são consecutivos nessa ordem. Uma ordem desse tipo é denominada ordem fortemente consistente de precedência. A Figura 2(a) ilustra um grafo que é 2-FPP e, na Figura 2(b), é ilustrado um grafo que é 2-fino próprio mas não é 2-FPP com relação à bipartição escolhida, problema considerado na Seção 2. Nessas figuras, as partes da bipartição de $V(G)$ estão sendo representadas por cores distintas. Além disso, a ordem fortemente consistente sendo considerada em ambas as figuras é $s=a, b, c, a^{\prime}, b^{\prime}, c^{\prime}$.

Em [Oliveira et al. 2018], foi apresentado uma caracterização e um algoritmo de reconhecimento eficiente para grafos 2-FPP, sendo que o último pode ser generalizado para grafos $k$-FPP, com $k$ fixo. Neste trabalho, estendemos esses resultados apresentando uma prova de NP-completude para o problema de reconhecimento de grafos $k$-FPP, com $k$ variável (parte da entrada), e uma caracterização para essa classe de grafos.

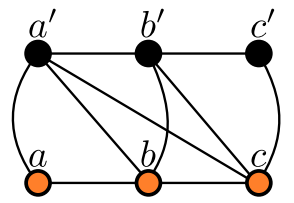

(a)

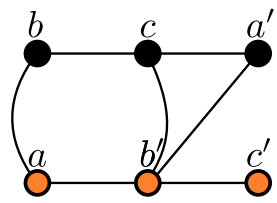

(b)

Figura 2. Grafos (a) 2-FPP e (b) 2-fino próprio não 2-FPP para tal bipartição.

\section{Reconhecimento de grafos $k$-FPP}

Nesta seção, será apresentada uma prova de NP-completude para o problema de reconhecimento de grafos $k$-FPP, $k$ arbitrário, definido a seguir.

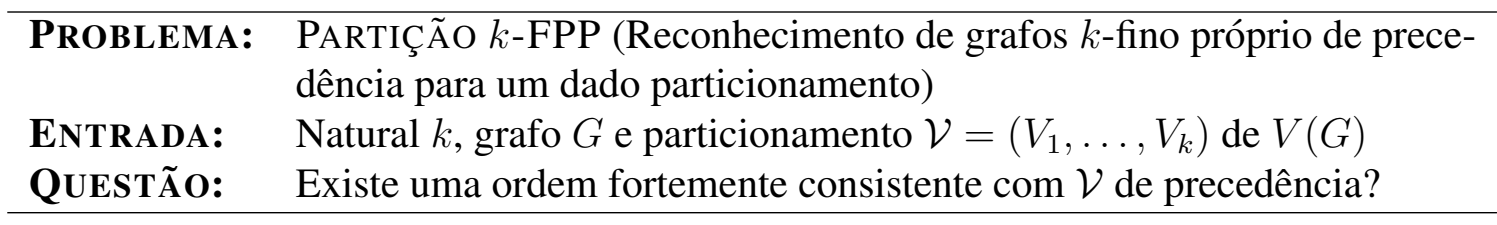

A prova de que o problema PARTIÇÃo $k$-FPP é NP-completo é obtida a partir de uma redução do problema NOT ALL EQUAL 3-SAT, que é NP-completo [Schaefer 1978]. Teorema 1. O problema PARTIÇÃO $k$-FPP é NP-completo, mesmo se o tamanho de cada parte da partição for no máximo 2. 
Ideia da demonstração. Uma ordem fortemente consistente de precedência pode ser verificada em tempo polinomial. Logo, esse problema está em NP. Dada uma instância $\varphi$ de NOT ALL EQUAL 3-SAT, definimos um grafo $G$ e um particionamento de $V(G)$ no qual cada parte tem tamanho no máximo dois. O grafo $G$ é construído como segue.

Para cada variável $x_{i}$ da cláusula $\mathcal{C}_{j}$, crie a parte $X_{i j}=\left\{x_{i j}^{T}, x_{i j}^{F}\right\}$. Ademais, para cada variável $x_{i}$, crie as partes $X_{i}^{T}=\left\{x_{i}^{T}\right\}$ e $X_{i}^{F}=\left\{x_{i}^{F}\right\}$. As arestas entre esses vértices são $\left(x_{i}^{T}, x_{i j}^{T}\right)$ e $\left(x_{i}^{F}, x_{i j}^{F}\right)$ para cada $i, j$ tais que a variável $x_{i}$ apareça na cláusula $\mathcal{C}_{j}$.

No que segue, se o $k$-ésimo literal $\ell_{i j}$ de $\mathcal{C}_{j}$ for a variável $x_{i}$ (resp. $\neg x_{i}$ ), denotamos por $O_{i j}$ o conjunto ordenado $\left\{x_{i j}^{F}, x_{i j}^{T}\right\}$ (resp. $\left\{x_{i j}^{T}, x_{i j}^{F}\right\}$ ). Dado um conjunto ordenado $C$ com dois vértices, denotamos por $C^{1}$ e $C^{2}$ o primeiro e segundo elementos de $C$, respectivamente. Para cada cláusula $\mathcal{C}_{j}=\ell_{1 j} \vee \ell_{2 j} \vee \ell_{3 j}$, serão adicionadas como partes, os conjuntos ordenados com dois elementos $Y_{1 j}, Y_{2 j}$ e $Y_{3 j}$, e as arestas $\left(O_{1 j}^{2}, Y_{1 j}^{1}\right),\left(O_{1 j}^{1}, Y_{2 j}^{1}\right)$, $\left(O_{1 j}^{2}, Y_{2 j}^{1}\right),\left(O_{2 j}^{1}, Y_{1 j}^{2}\right),\left(O_{2 j}^{1}, Y_{1 j}^{1}\right),\left(O_{2 j}^{2}, Y_{2 j}^{1}\right),\left(O_{2 j}^{2}, Y_{2 j}^{2}\right),\left(O_{2 j}^{1}, Y_{3 j}^{1}\right),\left(O_{2 j}^{1}, Y_{3 j}^{2}\right),\left(O_{3 j}^{1}, Y_{1 j}^{2}\right)$, $\left(O_{3 j}^{2}, Y_{1 j}^{2}\right),\left(O_{3 j}^{1}, Y_{2 j}^{2}\right),\left(O_{3 j}^{2}, Y_{2 j}^{2}\right),\left(O_{3 j}^{2}, Y_{3 j}^{1}\right),\left(O_{3 j}^{2}, Y_{3 j}^{2}\right)$.

É possível mostrar que existe uma ordem fortemente consistente de precedência para $V(G)$ se, e somente se, a atribuição $x_{i}=\left(x_{i}^{F}<x_{i}^{T}\right)$ (isto é, $x_{i}$ é verdade se $x_{i}^{F}$ preceder $x_{i}^{T}$ na ordem em questão e $x_{i}$ é falso caso contrário) satisfizer $\varphi$. A Figura 3 mostra a instância do problema PARTIÇÃo $k$-FPP construída a partir de uma dada instância $\varphi=\left\{\left(x_{1} \vee x_{2} \vee \neg x_{3}\right)\right\}$ do problema Not ALL EQUAL 3-SAT. Note que, a sequência $s=x_{1}^{F}, x_{2}^{F}, x_{3}^{F}, x_{11}^{F}, x_{11}^{T}, Y_{11}^{1}, Y_{11}^{2}, Y_{31}^{1}, Y_{31}^{2}, x_{21}^{F}, x_{21}^{T}, Y_{21}^{1}, Y_{21}^{2}, x_{31}^{F}, x_{31}^{T}, x_{1}^{T}, x_{2}^{T}, x_{3}^{T}$, relacionada à atribuição de verdade $x_{1}=x_{2}=x_{3}=T$, é uma possível solução para $(G, \varphi)$.

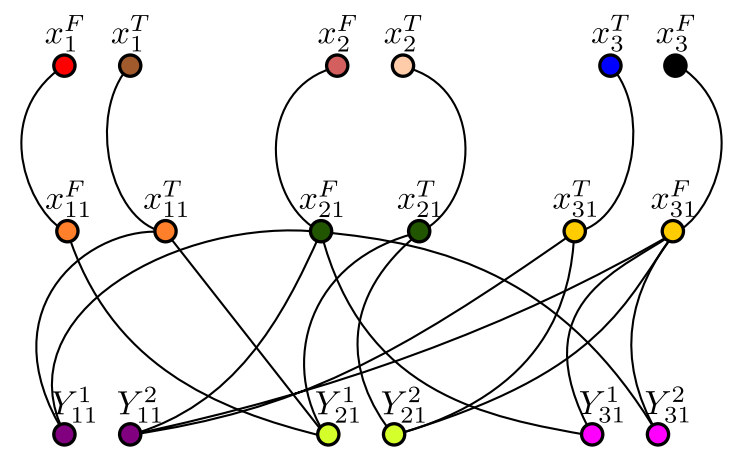

Figura 3. Instância do problema PARTIÇÃo $k$-FPP construída a partir da instância $\varphi=\left\{\left(x_{1} \vee x_{2} \vee \neg x_{3}\right)\right\}$ do problema NOT ALL EQUAL 3-SAT.

\section{Caracterização de grafos $\boldsymbol{k}$-fino próprio de precedência}

Seja $s=v_{1}, v_{2}, \ldots, v_{n^{\prime}}$ uma sequência de um subconjunto $V^{\prime} \subseteq V(G)$. Definimos $V(s)=V^{\prime}$ e $s^{-1}$ como a sequência reversa de $s$, ou seja, $s^{-1}=v_{n^{\prime}}, v_{n^{\prime}-1}, \ldots, v_{1}$.

Um grafo $G$ é dito de divisão se existir um biparticionamento $(X, Y)$ do seu conjunto de vértices tal que o grafo induzido por $X$ é uma clique e o grafo induzido por $Y$ é um conjunto independente. Um grafo de divisão $G$ é um grafo de limiar se existir uma ordem total para $X$ (resp. $Y$ ) tal que seus vértices estejam ordenados por ordem de inclusão de suas vizinhanças fechadas (resp. abertas). Uma ordem desse tipo é denominada de ordem de limiar de $X$ (resp. $Y$ ) em $G$, ou simplesmente ordem de limiar de $G$.

Seja $G$ um grafo e $(X, Y)$ um biparticionamento de $V(G)$. Definimos o grafo de divisão $S_{G}(X, Y)$ como o grafo obtido a partir de $G$ através da adição e remoção de 
arestas necessárias de tal modo que $X$ se torne uma clique e $Y$ um conjunto independente. Seja $s=s_{1} s_{2}$ ( $s$ consiste na concatenação das sequências $s_{1}$ e $s_{2}$ ) uma ordem total de $V(G)$. Dizemos que $\left(s_{1}, s_{2}\right)$ é compatível com $G$ se $s_{1}$ e $s_{2}$ forem ordens canônicas próprias de $G\left[V\left(s_{1}\right)\right]$ e $G\left[V\left(s_{2}\right)\right]$, respectivamente, e se $s_{1}$ e $s_{2}^{-1}$ forem ordens de limiar de $S_{G}\left(V\left(s_{1}\right), V\left(s_{2}\right)\right)$.

Teorema 2. Seja $G$ um grafo. Para todo $k \geq 2$, Gé $k$-FPP se, e somente se, existir um particionamento $\mathcal{V}=\left(V_{1}, \ldots, V_{k}\right)$ e uma ordem total $s=s_{1}, \ldots, s_{k}$ de $V(G)$ tal que para todo $1 \leq i<j \leq k,\left(s_{i}, s_{j}\right)$ é compatível com $G\left[V\left(s_{i}\right) \cup V\left(s_{j}\right)\right]$.

Demonstração. Primeiro, suponha $k=2$. Considere que $G$ é 2-FPP com relação a $\mathcal{V}=\left(V_{1}, V_{2}\right)$. Seja $s=s_{1} s_{2}$ uma ordem fortemente consistente de precedência de $G, V_{1}=V\left(s_{1}\right)$ e $V_{2}=V\left(s_{2}\right)$. Portanto $s_{1}$ (resp. $s_{2}$ ) é uma ordem canônica própria de $G\left[V\left(s_{1}\right)\right]$ (resp. $G\left[V\left(s_{2}\right)\right]$ ). Suponha, por absurdo, que $s_{1}$ não seja uma ordem de limiar de $S_{G}\left(V_{1}, V_{2}\right)$. Nesse caso, existem $u, v \in V_{1}$ e $w \in V_{2}$, com $u<v$ em $s_{1}$, tais que $w \in N[u]$ e $w \notin N[v]$. Como $u<v<w$ em $s$, existe uma contradição com o fato de $s$ ser uma ordem fortemente consistente de precedência de $V(G)$. Agora, suponha, por absurdo, que $s_{2}^{-1}$ não seja uma ordem de limiar de $S_{G}\left(V_{1}, V_{2}\right)$. Consequentemente, existem $u, v \in V_{2}$ e $w \in V_{1}$, com $u<v$ em $s_{2}$, tais que $w \in N(v)$ e $w \notin N(u)$, contradizendo o fato de $s$ ser uma ordem fortemente consistente de precedência, já que $w<u<v$ em $s$. Logo, $\left(s_{1}, s_{2}\right)$ é compatível com $G$.

Por outro lado, considere que existam ordens canônicas próprias $s_{1}$ e $s_{2}$ de $V_{1}$ e $V_{2}$, respectivamente, tais que $\left(s_{1}, s_{2}\right)$ é compatível com $G$. Isto é, $s_{1}$ e $s_{2}^{-1}$ são ordens de limiar de $S_{G}\left(V_{1}, V_{2}\right)$. A seguir, é provado que $s=s_{1} s_{2}$ é uma ordem fortemente consistente de precedência de $G$ considerando o biparticionamento $\left(V_{1}, V_{2}\right)$. Suponha que a afirmação anterior não seja verdade. Isto implica que existem (i) $u, v \in V_{1}, w \in V_{2} \operatorname{com} u<v$ em $s$ tais que $(u, w) \in E(G),(v, w) \notin E(G)$, ou (ii) $u, v \in V_{2}, w \in V_{1}$ com $u<v$ em $s_{2}$ tais que $(v, w) \in E(G),(u, w) \notin E(G)$. No caso (i) (resp. (ii)), tem-se uma contradição com o fato de $s_{1}$ (resp. $s_{2}$ ) ser uma ordem de limiar de $S_{G}\left(V_{1}, V_{2}\right)$.

Para $k>2$, suponha que exista um particionamento $\mathcal{V}=\left(V_{1}, \ldots, V_{k}\right)$ e uma ordem total $s=s_{1} \ldots s_{k}$ de $V(G)$ tal que, para todo $1 \leq i \leq k, V\left(s_{i}\right) \in \mathcal{V}$. Note que $s$ é uma ordem fortemente consistente de precedência se, e somente se, $s_{i} s_{j}$ for uma ordem fortemente consistente de precedência de $G\left[V_{i} \cup V_{j}\right]$ com relação ao biparticionamento $\left(V_{i}, V_{j}\right)$, para todo $1 \leq i<j \leq k$. A partir da prova do caso $k=2$, tem-se que a ordem $s_{i} s_{j}$ é uma ordem fortemente consistente de precedência se, e somente se, $\left(s_{i}, s_{j}\right)$ for compartível com $G\left[V\left(s_{i}\right) \cup V\left(s_{j}\right)\right]$.

\section{Referências}

Bonomo, F. and de Estrada, D. (2019). On the thinness and proper thinness of a graph. Discrete Applied Mathematics, 261:78-92.

Oliveira, F. S., Sampaio Jr., M. S., and Szwarcfiter, J. L. (2018). Sobre finura própria de grafos. In Anais do III Encontro de Teoria da Computação. SBC.

Roberts, F. (1969). Indifference graphs. In Harary, F., editor, Proof Techniques in Graph Theory, pages 139-146. Academic Press, New York.

Schaefer, T. J. (1978). The complexity of satisfiability problems. In Proceedings of the Tenth Annual ACM Symposium on Theory of Computing, STOC '78, pages 216-226. 